\title{
On the identification of some Entamoeba species - response to Ponce-Gordo and Martínez-Díaz
}

The recent comments by Ponce-Gordo \& Martínez-Díaz (2007) on our paper in IJSEM (Clark et al., 2006) are unfortunate as they are based on a misreading of the text and a misunderstanding of our results.

The authors are entitled to their opinion that we are not justified in attributing the name Entamoeba equi to our horse isolate and can use Entamoeba sp. if they prefer. However, we take issue with their reasoning. Their criticism of our citation comes from a misreading - the citation was for the medium, not the amoeba. Describing the morphology of the culture trophozoites would in any case have been useless as it is well known that trophozoite size in culture need not reflect size in vivo. Species of the genus Colpodella are aerobic organisms while Entamoeba species are anaerobic, so they cannot both derive from contamination. We did not see cysts, but we did show that the small subunit rRNA gene sequence of the Entamoeba clusters strongly with the 4-nucleated cyst clade in phylogenetic trees. Since the sequence represents a distinct lineage with no specific relatives and is from a unique host, and since $E$. equi is the only valid species name for a 4-nucleated cyst-producing Entamoeba species in horses, we feel justified in using it for this organism.

We were well aware of the genetic differences between the authors' Entamoeba struthionis (Ponce-Gordo et al., 2004) and the three other uninucleated Entamoeba groups. However, like Verweij et al. (2001), we concluded that the comparatively small sequence divergence observed among these four genetic groups of uninucleated Entamoeba is not adequate justification on its own for separating them into distinct species and that the name Entamoeba polecki has precedence. These four groups, all of which have been found in humans, are the variants of E. polecki to which we refer. The authors have suggested that the amoebae from three humans (Verweij et al., 2001) and a pig that gave a match to their sequence from ostrich isolates all result from 'spurious parasitosis', by which they presumably mean ingested cysts passing through without establishing an infection. It is difficult to imagine a situation in which humans could ingest sufficient ostrich faeces to produce Entamoeba cysts in their stools that would be easily detectable by microscopy, yet this is how all these infections were originally identified. Four additional human samples containing this particular type of uninucleated Entamoeba have since been found and, in one case, both trophozoites and cysts were observed in the sodium acetate-acetic acid-formalin (SAF)-fixed portion of the faeces (J. J. Verweij, personal communication). It is notable that the nuclear morphology of these organisms is variable, even though the sequences are identical.

The studies by Blessmann et al. (Blessmann et al., 2002, 2003) did not ignore uninucleated cyst infections, the data were simply not reported until now. Those publications focussed on Entamoeba histolytica and so the data on uninucleates were not relevant. However, the pig samples containing Entamoeba suis were acquired during those studies, which is why the papers were cited. The prevalence of human uninucleated Entamoeba infections in Viet Nam was reported in our IJSEM paper (3.5\%) and the relevant sequences matched the database entry for Entamoeba chattoni (unpublished data).

In summary, we feel the criticisms of Ponce-Gordo and Martínez-Díaz are unwarranted and we stand by our conclusions.

\author{
C. Graham Clark, ${ }^{1}$ Jeffrey J. Windsor ${ }^{2}$ \\ and Egbert Tannich ${ }^{3}$ \\ ${ }^{1}$ Department of Infectious and Tropical \\ Diseases, London School of Hygiene \\ and Tropical Medicine, London WC1E \\ 7HT, UK \\ ${ }^{2}$ National Public Health Service for \\ Wales Aberystwyth, Bronglais Hospital, \\ Aberystwyth, Ceredigion SY23 1ER, \\ Wales, UK \\ ${ }^{3}$ Department of Molecular Parasitology, \\ Bernhard Nocht Institute for Tropical \\ Medicine, D-20359 Hamburg, Germany
}

\section{Correspondence: C. Graham Clark (graham.clark@lshtm.ac.uk)}

\begin{abstract}
Blessmann, J., Van Linh, P., Nu, P. A., Thi, H. D., Muller-Myhsok, B., Buss, H. \& Tannich, E. (2002). Epidemiology of amebiasis in a region of high incidence of amebic liver abscess in central Vietnam. Am J Trop Med Hyg 66, 578-583.

Blessmann, J., Ali, I. K. M., Nu, P. A. T., Dinh, B. T., Ngo Viet, T. Q., Van, A. L., Clark, C. G. \& Tannich, E. (2003). Longitudinal study of intestinal Entamoeba histolytica infections in asymptomatic adult carriers. J Clin Microbiol 41, 4745-4750.
\end{abstract}

Clark, C. G., Kaffashian, F., Tawari, B., Windsor, J. J., Twigg-Flesner, A., Davies-Morel, M. C. G., Blessmann, J., Ebert, F., Peschel, B. \& other authors (2006). New insights into the phylogeny of Entamoeba species provided by analysis of four new small-subunit rRNA genes. Int J Syst Evol Microbiol 56, 2235-2239.

Ponce-Gordo, F. \& Martínez-Díaz, R. A. (2007). On the identification of some Entamoeba species. Comments on a recent paper. Int J Syst Evol Microbiol 57, 207.

Ponce-Gordo, F., Martínez-Díaz, R. A. \& Herrera, S. (2004). Entamoeba struthionis n.sp. (Sarcomastigophora: Endamoebidae) from ostriches (Struthio camelus). Vet Parasitol 119, 327-335.

Verweij, J. J., Polderman, A. M. \& Clark, C. G. (2001). Genetic variation among human isolates of uninucleated cyst-producing Entamoeba species. J Clin Microbiol 39, 1644-1646. 